\title{
Musik, Gesellschaftskritik und utopische Vision in Menschheitsdämmerung und Geist der Utopie
}

\section{Music, Social Critique and Utopian Vision in The Spirit of Utopia and Dawn of Humanit}

\author{
Nina CEMILOĞLU'
}

${ }^{1}$ Assist. Prof. Dr., Yeditepe University, Faculty of Arts and Sciences, English Language and Literature Department Istanbul, Turkey

ORCID: N.C. 0000-0002-3977-7314

\section{Corresponding author:}

Nina CEMILOĞLU,

Yeditepe Üniversitesi, Fen-Edebiyat Fakültesi, İngiliz Dili ve Edebiyatı Bölümü, İnönü Mah. Kayıșdağı Cad. 326 A, 26 Ağustos Yerleşimi, 34755 Ataşehir, İstanbul, Turkey

E-mail: nina.cemiloglu@yeditepe.edu.tr

Submitted: 07.12 .2018

Revision Requested: 15.04.2019

Last Revision Received: 24.04.2019

Accepted: 17.04.2019

Citation: Cemiloglu, N. (2019). Musik, gesellschaftskritik und utopische vision in menschheitsdämmerung und geist der utopie. Litera, 29(1), 95-112.

https://doi.org/10.26650/LITERA2018-0012

\section{ABSTRACT (DEUTSCH)}

Dieser Artikel befasst sich mit zwei wichtigen Texten des deutschen Expressionismus: Ernst Blochs Geist der Utopie (1918/1923) und Kurt Pinthus'Menschheitsdämmerung (1919). Geist der Utopie gilt als wichtigstes philosophisches Frühwerk Ernst Blochs. Menschheitsdämmerung wird als bedeutendste und einflussreichste Anthologie deutscher expressionistischer Dichtung angesehen. Ihr Herausgeber ist Kurt Pinthus. Ziel dieses Artikels ist es, thematische Bezugspunkte zwischen Geist der Utopie und Menschheitsdämmerung aufzuzeigen. Dieser Artikel gliedert sich in drei Teile: Einleitung, Hauptteil und Schlussbetrachtung. Der Haupteil ist in drei Teile untergliedert: Der erste Teil beschäftigt sich mit der Konzipierung von Menschheitsdämmerung als Symphonie und mit Blochs Auffassung von Musik. Besondere Aufmerksamkeit wird der Bedeutung der sogenannten Humanitäts-Melodie von Ludwig van Beethoven zuteil. Der zweite Teil befasst sich mit Gesellschaftskritik in Geist der Utopie und in ausgewählten Gedichten der Menschheitsdämmerung. Ausgewählt wurden die folgenden Gedichte: Städter und Das Herz von Alfred Wolfenstein, Der Krieg von Georg Heym, und Arbeiter! von Karl Otten. Besondere Aufmerksamkeit gilt den Problemen Einsamkeit, Isolation, Entfremdung, Ausbeutung und Krieg. Der dritte Teil unternimmt den Versuch, utopische Vision in ausgewählten Gedichten der Menschheitsdämmerung und in Geist der Utopie aufzuzeigen. Es wurden die folgenden Gedichte ausgewählt: Mensch stehe auf von Johannes R. Becher, Gethsemane von Kurt Heynicke, und Zwiegespräch von Ernst Stadler. Im Mittelpunkt stehen die Themen Brüderschaft, Aussöhnung, Gemeinschaft, Erlösung, kulturelles Erbe und Heimat.

Schlüsselwörter: Literatur, Philosophie, Musik, Utopie, deutsche expressionistische Dichtung

\section{ABSTRACT}

This article is about two important texts of German expressionism: Ernst Bloch's The Spirit of Utopia (1918/1923) and Kurt Pinthus' Dawn of Humanity (1919). The Spirit of Utopia is considered Ernst Bloch's most important early philosophical work. Dawn of Humanity is regarded as the most representative and influential anthology of German expressionist poetry. Its editor is Kurt Pinthus. The aim of this article is to explore thematic affinities between The Spirit of Utopia and Dawn of Humanity. This article is divided into three parts: Introduction, main part and conclusion. The main part is subdivided into three sections: The first section deals with the conception of Dawn of Humanity as a symphony and with Bloch's views on music. Special attention is given to the importance of the socalled humanity melody by Ludwig van Beethoven for Bloch and Pinthus. The second section focuses on social critique in The Spirit of Utopia and selected poems of Dawn of Humanity. The poems selected for discussion are: Alfred Wolfenstein's City Dwellers and The Heart, Georg Heym's The War, and Karl Otten's Worker!. Special attention is given to the problems of isolation, alienation, exploitation and war. The third section attempts to trace utopian vision in selected poems of Dawn of Humanity and in The Spirit of Utopia. The poems selected for discussion are: Johannes R. Becher's Human, Rise, Kurt Heynicke's Gethsemane and Ernst Stadler's Dialogue. This section pays special attention to the themes of brotherhood, reconciliation, communion, redemption, the cultural heritage and home. Keywords: Literature, philosophy, music, utopia, German expressionist poetry 


\section{EXTENDED ABSTRACT}

This article is about two important texts of German expressionism: The Spirit of Utopia and Dawn of Humanity. The Spirit of Utopia was written by Ernst Bloch (18851977), a German philosopher associated with the Frankfurt School of Critical Theory. The Spirit of Utopia was written between 1914 and 1917 and published in 1918. It was then revised by Bloch and appeared in a second edition in 1923. The Spirit of Utopia is considered Bloch's first important philosophical work. It had a profound and lasting impact on many Frankfurt School thinkers, such as Theodor W. Adorno and Walter Benjamin (the latter was not a member, but, like Bloch, closely associated with the Frankfurt School). The Spirit of Utopia contains all the philosophical questions which preoccupied Bloch throughout his life and which found their most elaborate expression in his main philosophical work The Principle of Hope (1959). Dawn of Humanity is an anthology of German expressionist poetry. It was edited by Kurt Pinthus (1886-1975) and first published in 1919. Its complete title is Dawn of Humanity: Symphony of Most Recent Poetry. It is considered the most representative and influential anthology of German expressionist poetry. Its poems were written between 1910 and 1920. Dawn of Humanity contains poems by both well-known writers, such as Else Lasker-Schüler, Franz Werfel, Gottfried Benn, Johannes R. Becher and Georg Trakl, and by lesser known writers, such as Alfred Wolfenstein, Karl Otten, Kurt Heynicke and Ernst Stadler. As editor and critic, Pinthus was an important and influential advocate of German expressionist literature. Throughout his life he was committed to reconstructing and making accessible to the German reading public the life and work of writers who were persecuted by the Nazis from 1933 to 1945 and whose books were suppressed and destroyed by them.

The aim of this article is to explore thematic affinities between The Spirit of Utopia and Dawn of Humanity. This article is divided into three parts: Introduction, main part, and conclusion. The main part is subdivided into three sections: 1. Dawn of Humanity as a symphony and Bloch's philosophy of music, 2. Social critique in The Spirit of Utopia and selected poems of Dawn of Humanity, 3. Utopian vision in selected poems of Dawn of Humanity and The Spirit of Utopia. The first section deals with the conception of Dawn of Humanity as a symphony and with Bloch's views on music. Special attention is given to the importance of the so-called humanity melody by Ludwig van Beethoven for Bloch and Pinthus. This section mainly refers to the following texts: Zuvor (Before) and Nach 40 Jahren (After 40 Years) by Kurt Pinthus and 
Philosophie der Musik (The Philosophy of Music) and Zur Theorie der Musik (About the Theory of Music) by Ernst Bloch. The former texts are prefaces to Dawn of Humanity and were published in 1919 and 1959 respectively. The latter texts constitute two chapters of The Spirit of Utopia. The second section of this article focuses on social critique in The Spirit of Utopia and selected poems of Dawn of Humanity. The poems selected for discussion are: Alfred Wolfenstein's Städter (City Dwellers) and Das Herz (The Heart), Georg Heym's Der Krieg (The War) and Karl Otten's Arbeiter! (Worker!). Special attention is given to the problems of loneliness, isolation, alienation, exploitation and war. The third section attempts to trace utopian vision in selected poems of Dawn of Humanity and The Spirit of Utopia. The poems selected for discussion are: Johannes R. Becher's Mensch stehe auf (Human, Rise), Kurt Heynicke's Gethsemane and Ernst Stadler's Zwiegespräch (Dialogue). The focus of this section is on the concepts of brotherhood, reconciliation, communion, redemption, the cultural heritage and home. 


\section{Einleitung}

Geist der Utopie gilt als wichtigstes philosophisches Frühwerk Ernst Blochs. Es entstand zwischen 1915 und 1917 und wurde erstmals im Jahr 1918 veröffentlicht. Eine überarbeitete Fassung erschien 1923. In einem Brief an Ernst Schoen aus dem Jahr 1919 bezeichnete Walter Benjamin (1978, S. 219) Geist der Utopie als das einzige in jenem Jahr erschienene Buch, an dem er sich „als an einer wahrhaft gleichzeitigen und zeitgenössischen Äußerung messen" könne. Benjamin (1978, S. 219) lobt das Buch als herausragend, denn „der Verfasser steht allein und steht philosophisch für diese Sache ein, während fast alles, was wir, von Gleichzeitigen, heute, philosophisch Gedachtes, lesen sich anlehnt, sich vermischt und nirgends an dem Punkte seiner Verantwortung zu fassen ist". Ernst Bloch $(1975$, S. 2) bemerkte im Rückblick, er habe Geist der Utopie ,mit viel Beethoven außer Hegel im Kopf, nicht ohne Berührung mit dem Expressionismus des Blauen Reiters" geschrieben. In der berühmten Expressionismusdebatte, in der viele Kritiker den Expressionismus als Wegbereiter der nationalsozialistischen Ideologie und Schreckensherrschaft verurteilten, verteidigte Bloch (1938/ 1969, S. 57) expressionistische Kunst als zukunftsweisend, weil sie den Menschen in den Mittelpunkt stellt:

Der Expressionismus hatte gerade in dem Bedeutung, worin ihn Ziegler verurteilt: er hat den Schlendrian und Akademismus zersetzt, zu dem die 'Kunstwerke' verkommen waren. Er hat statt der ewigen 'Formanalyse' am objet d'art auf den Menschen und seinen Inhalt verwiesen, der zum möglichst echten Ausdruck drängt. (Bloch, 1938/ 1969, S. 57)

Menschheitsdämmerung gilt als die bedeutendste und einflussreichste Anthologie deutscher expressionistischer Dichtung. Die von Kurt Pinthus herausgegebene Lyrikanthologie erschien erstmals im Jahr 1919. Pinthus (1959/ 1996, S. 15) hat hervorgehoben, dass der Expressionismus, wie der Humanismus, sich unmittelbar mit dem Menschen beschäftigt und sich unmittelbar an den Menschen wendet. Dies trifft auch auf Menschheitsdämmerung zu. Pinthus (1959/ 1996, S. 15) bezeichnet die deutschen expressionistischen Dichter als "enttäuschte Humanisten" und stellt die These auf, dass „die utopischen Forderungen des Expressionismus nicht von Marx, sondern vom Humanismus herstammen“. Pinthus (1919/ 1996, S. 22) definiert Dichtung als „das Barometer seelischer Zustände, der Bewegung und Bewegtheit der Menschheit", "das Auf, Ab und Empor des Denkens und Sehnens“. Die deutschen 
expressionistischen Dichter charakterisiert Pinthus (1919/ 1996, S. 32) als inspiriert von der "Hoffnung auf den Menschen und de[n] Glaube[n] an die Utopie“. Diese Formulierung trifft auch auf Bloch zu. Im Mittelpunkt seines Denkens steht der Mensch und sein Verhältnis zur Welt. Kunst nimmt eine zentralen Platz in Blochs utopischer Philosophie ein. Er betrachtet Utopie nicht als literarisches Genre oder politisches Programm, sondern als ontologische Kategorie. Bloch zufolge ist alles utopisch: Der Mensch, die Welt und die Kunst. Er sieht Sehnsucht und Verlangen als wichtigste Eigenschaften des Menschen. Das utopische Wesen des Menschen manifestiert sich in Tagträumen und Kunstwerken. Anders als Freud misst Bloch dem Tagtraum eine grössere Bedeutung zu als dem Nachttraum. Bloch (1923/ 1985, S. 186) hielt den Tagtraum für erforschenswerter als den Nachttraum, weil der Tagtraum ein ",noch nicht bewußtes Wissen", das sich auf noch Ungeschehenes in der Zukunft bezieht, enthält. Hingegen ist der Nachttraum „nur ein blosses sich Erinnern dessen, was schon war" (Bloch 1923/ 1985, S. 186). Tagträume und Kunstwerke besitzen „die Würde der Erkenntnis, der spezifischen Erkenntnis einer ästhetischen Wirklichkeit und des utopisch-möglichen Ideegebots ihrer Sphäre" (Bloch 1923/ 1985, S. 150). Bloch betrachtet den Tagtraum und das Kunstwerk als kognitiv: Beide enthalten utopisches Wissen. Dieses Wissen ist jedoch nicht konkret und eindeutig. Bloch beschreibt es mit dem Begriff "Vorschein". Er hat darauf hingewiesen, dass auch Kunstwerke aus vergangenen Epochen „Vorschein" enthalten, denn "das utopische Bewusstsein [legt] auch noch das Heraufkommende im alten frei... Es entdeckt die wirkliche Tiefe - in der Höhe, nämlich in der des hellsten Bewusstseins, wo noch helleres dämmert" (Bloch 1959/ 1985, S. 161).

Der Begriff „Vorschein“ und die häufige Verwendung von Wörtern wie „dämmern“ und „Dämmerung" in Blochs Werken verweisen auf die wichtige Rolle, die Licht in Blochs Denken spielt. Bloch (1923/ 1985, S. 13) bezeichnet die menschliche Sehnsucht nach einem besseren Leben als „Licht", das „in uns allein brennt". Desweiteren hebt er die Bedeutung zweier mythischer Figuren, die mit Licht in Verbindung stehen, für die Menschheit hervor: Prometheus und Luzifer. Bloch (1959/ 1985, S. 1150) bezeichnet Prometheus als „,der Alleswoller, der Allesträumer, der Lichtrebell, der den Menschen das Feuer gebracht hat, ja der das Feuer selber ist". Luzifer wird von Bloch (1959/ 1985, S. 1239) als „Bewußtseinsmacher, Lichtschlager, Weltveränderer“ beschrieben. Bloch war davon überzeugt, dass die Welt vom Menschen verändert werden kann. Auch Menschheitsdämmerung hat Weltveränderung im Sinn. Jürgen Serke (1983, S. 396) hat darauf hingewiesen, dass sich die expressionistische Literatur als "Revolution, als 
Aufbruch, Erhebung und Wandlung" auffasste. Wie der Titel Menschheitsdämmerung andeutet, sind die in ihr enthaltenen Gedichte Ausdruck der Überzeugung, dass ein Zeitalter zu Ende geht und ein neues anfängt. Die expressionistischen Dichter kämpften gegen "die Menschheit der zu Ende gehenden Epoche und [für die] Vorbereitung [...] neuer, besserer Menschheit" (Pinthus 1919/ 1996, S. 23). Die Gedichte der Menschheitsdämmerung wenden sich gegen eine menschenfeindliche Ideologie und Gesellschaftsordnung und fordern ein menschgemässes Leben. Sie enthalten Gesellschaftskritik und utopische Vision. Dies trifft auch auf Geist der Utopie zu. Ausserdem spielt Musik eine wichtige Rolle in beiden Werken.

\section{Menschheitsdämmerung als Symphonie und Blochs Philosophie der Musik}

Menschheitsdämmerung enthält Gedichte aus den Jahren 1910-1920. Der vollständige Titel der von Kurt Pinthus herausgegebenen Lyrikanthologie lautet Menschheitsdämmerung - Symphonie jüngster Dichtung. Pinthus ordnete die von ihm ausgewählten expressionistischen Gedichte nicht alphabetisch oder chronologisch, sondern symphonisch. Pinthus (1959/ 1996, S. 7) erklärte, es sei ihm wichtig gewesen "die Dichter nicht in chronologischer oder alphabetischer Folge aufziehen zu lassen, sondern ihre Gedichte nach großen Hauptmotiven, kleinen und kleinsten Motiven zu ordnen, zu verflechten und zu komponieren, ähnlich dem Aufbau einer Symphonie in vier Sätzen". Pinthus (1919/ 1996, S. 22) betonte, dass die symphonische Anordnung der Gedichte nicht auf ein vertikales und kausales Betrachten der versammelten Gedichte ziele, sondern auf ein horizontales und simultanes Hineinhören:

Man horche in die Dichtung unserer Zeit .., man horche quer durch, man blicke rund herum,... nicht vertikal, nicht nacheinander, sondern horizontal; man scheide nicht das Aufeinanderfolgende auseinander, sondern man höre zusammen, zugleich, simultan. Man höre den Zusammenklang dichtender Stimmen: man höre symphonisch.

So wie Pinthus es ablehnte, seine Anthologie alphabetisch oder chronologisch zu ordnen, lehnte er es ab, in ihr Musterbeispiele expressionistischer Lyrik oder nur besonders schöne expressionistische Gedichte zu präsentieren (1919/ 1996, S. 22). Tatsächlich waren die expressionistischen Dichter weniger daran interessiert, schöne Gedichte zu schreiben, als daran, auf politische, ethische und soziale Probleme 
aufmerksam zu machen. Jürgen Serke $(1983$, S. 396) bezeichnet die Werke deutscher expressionistischer Schriftsteller als weit über alle ästhetischen Kriterien hinausgehend, als "Moderne in ihrer radikalsten Form“. Pinthus (1919/ 1996, S. 30) hat darauf hingewiesen, dass "die Qualität dieser Dichtung in ihrer Intensität beruht":

Niemals in der Weltdichtung scholl so laut, zerreissend und aufrüttelnd Schrei, Sturz und Sehnsucht einer Zeit, wie aus dem wilden Zuge dieser Vorläufer und Märtyrer, deren Herzen nicht von den romantischen Pfeilen des Amor oder Eros, sondern von den Peinigungen verdammter Jugend, verhaßter Gesellschaft, aufgezwungener Mordjahre durchbohrt wurden.

Zwei der vier Teile, in die Pinthus Menschheitsdämmerung gliederte, tragen die Überschriften „Sturz und Schrei” und „Aufruf und Empörung". Diese Teile enthalten Gedichte, die wie laute Stimmen "klagen, schreien, fluchen, rufen" (Pinthus 1919/ 1996, S. 31). Zwei weitere Teile der Anthologie tragen die Überschriften „Erweckung des Herzen" und "Liebe den Menschen". Pinthus (1919/ 1996, S. 23) nannte den ersten Teil ein „Andante des Zweifels und der Verzweiflung", den zweiten Teil ein „Moderato des erwachenden, erweckten Herzens", den dritten Teil ein „Furioso der Empörung" und den vierten Teil ein „Maestoso der menschenliebenden Menschheit".

In seinem Vorwort zur Neuauflage von Menschheitsdämmerung im Jahr 1959 erklärte Pinthus (S. 14), seine Konzipierung der Menschheitsdämmerung als Symphonie könne unbewusst von Beethovens sogenannter Humanitäts-Melodie inspiriert gewesen sein. Rückblickend bezeichnete Pinthus (1959/ 1996, S. 14) Beethovens sogenannte Humanitäts-Melodie als „,das messianische Hauptmotiv des Expressionismus". Beethoven komponierte diese Melodie anlässlich des Todes des aufklärerischen und volksnahen Kaisers Josef II. zu den Worten "Da stiegen die Menschen, die Menschen ans Licht" (Pinthus 1959/ 1996, S. 14). Beethoven liess die Humanitätsmelodie fünfzehn Jahre später noch einmal in Fidelio erklingen: In der Szene, in der Leonore ihren Geliebten befreit (Pinthus 1959/ 1996, S. 14). Bloch (1965/ 1985, S. 579) hat darauf aufmerksam gemacht, dass Leonore, als sie das Gesicht des Mannes, der sich ihr nähert, nicht klar erkennen kann, den Entschluss fasst, nicht nur ihren Geliebten zu befreien, sondern alle Gefangenen, d.h. symbolisch alle Menschen. Sowohl Bloch (1965/ 1985, S. 578) als auch Alfred Kazin (1976, S. 6) haben darauf hingewiesen, dass die Französische Revolution mit ihrer Forderung nach Freiheit, Gleichheit und Brüderlichkeit einen grossen Einfluss auf Beethoven gehabt hat. Kazin 
(1976, S. 1) bemerkt darüberhinaus (in einer stark an Bloch erinnernden Wendung), wie viel Zukunft Beethovens Musik enthalte. Bloch, der klassische Musik liebte, hebt immer wieder das besondere Genie Beethovens hervor. Bloch (1923/ 1985, S. 88) nennt Beethoven den "größten Erwählten des dynamischen, luziferischen Geistes" und beschreibt seine Musik folgendermassen:

Laut und rücksichtslos hebt sich der Schrei des Beethovenschen Subjekts heraus, dem nichts in dem scheinbaren Leben Genüge tut, das noch über dem höchsten Niveau jeder weltlich realen Umschließung steht, das gleich dem Genius der Musik selber nirgends in der Welt vorgebildet ist oder empfangen wird. Das durch alle Skalen der Leidenschaft und Phantastik hindurchfährt und doch stets wieder auf sich, auf die Sehnsucht nach dem Bruder und dem visionären Vater zurückgetrieben wird. Es ist hier eine Leidenschaft, das blo $\beta$ innere Leben, die geschlossene Stille der Innerlichkeit zu verlassen, die das Ich zu einem wahrhaft kosmischen Gebilde verwandelt, so hoch und so tief, da $\beta$ in ihm, ohne sich anzustoßen, Sonne, Mond und Sterne auf- und untergehen könnten, und der ganze Umkreis der Menschlichkeit seinen Platz findet. (Bloch 1923/ 1985, S. 87)

Bloch hört in Beethovens Musik utopische Sehnsucht und Vision. Bezeichnenderweise charakterisiert Bloch (1923/ 1985, S. 78) Fidelio nicht als Oper, sondern als Symphonie: „Der ausschweifend gehandhabte Ton, das Gewoge der Töne und der andauernde Zuschuß aus Spannung, Chaos und Schicksal schäumt zu einer überwiegend unmelodischen, rezitativisch melismatischen, motivisch thematischen, und sich im Ganzen rein symphonisch entwickelnden Musikart über". Bloch vergleicht die symphonische Musik Beethovens mit dem Chorgesang des Mittelalters. Ihm zufolge vereint nicht nur ein Chor, sondern auch ein Orchester menschliche Stimmen. Er vergleicht die Symphonie mit dem Chorgesang der mittelalterlichen christlichen Glaubensgemeinde. Dementsprechend bezeichnet Bloch (1923/ 1985, S. 79) die Symphonie als "ein anderes sich Versammeln" und als „eine andere Sehnsucht nach Organisation, einer Erdballsbreite, die die Menschen zusammenführt, die ihnen im Chorwerk tausend Stimmen schenkt, um nach dem Einen zu verlangen“. Menschheitsdämmerung ist eine Symphonie menschlicher Stimmen, die "nach dem edleren, menschlicheren Menschen [schreien]" (Pinthus 1919/ 1996, S. 23). Die Gedichte der Menschheitsdämmerung beinhalten Kritik am Zustand der Menschheit zu Beginn des 20. Jahrhunderts und utopische Vision einer menschgemässen Zukunft. 


\section{Gesellschaftskritik in Geist der Utopie und in ausgewählten Gedichten der Menschheitsdämmerung}

Bloch hat immer wieder auf die spirituelle Leere in der modernen kapitalistischen Gesellschaft hingewiesen. In Geist der Utopie (1923/ 1985, S. 11-12) bezeichnete Bloch sein Zeitalter als "geistlos und unchristlich" und als eingehüllt in "starre Verfinsterung". In einem später entstandenen Werk nannte Bloch (1971, S.64) die kapitalistische Gesellschaft "diese Öde, das frierende Unzuhause, mit kurzen Betäubungen und dahinter eine Finsternis". Bloch (1923/ 1985, S. 12) zufolge lebt der moderne Mensch „in völlig kreatürlicher Beschränktheit, irreligiöser Erloschenheit". In der kapitalistischen Gesellschaft besteht das Leben nur aus „Tanz ums Kalb“ (Bloch 1923/ 1985, S. 12). Die Folgen dieser Lebens- und Denkweise sind Egoismus, Gewissenlosigkeit und Phantasiearmut:

[W]ir haben keinen sozialistischen Gedanken. Sondern wir sind ärmer als die warmen Tiere geworden; wem nicht der Bauch, dem ist der Staat sein Gott, alles andere ist zum Spass und zur Unterhaltung herabgesunken. Wir haben Sehnsucht und kurzes Wissen, aber wenig Tat und, was deren Fehlen erklärt, keine Weite, keine Aussicht, keine Enden, keine innere Schwelle, geahnt überschritten, keinen Kern und kein sammelndes Gewissen des Überhaupt. (Bloch 1923/ 1985, S. 12-13)

Beim „Tanz ums Kalb" gibt es kein Miteinander oder Füreinander. In der kapitalistischen Hetze bleibt jeder für sich: einsam, isoliert und entfremdet. Einsamkeit, Isolation und Entfremdung sind zentrale Themen in Menschheitsdämmerung. Alfred Wolfensteins Gedicht Städter (Pinthus, 1996, S. 45-46) beschreibt Einsamkeit, Isolation und Entfremdung folgendermassen:

Nah wie Löcher eines Siebes stehn

Fenster beieinander, drängend fassen

Häuser sich so dicht an, dass die Straßen

Grau geschwollen wie Gewürgte sehn.

Ineinander dicht hineingehakt

Sitzen in den Trams die zwei Fassaden

Leute, wo die Blicke eng ausladen

Und Begierde ineinander ragt. 
Unsre Wände sind so dünn wie Haut,

$\mathrm{Da} \beta$ ein jeder teilnimmt, wenn ich weine,

Flüstern dringt hinüber wie Gegröhle:

Und wie stumm in abgeschloßner Höhle

Unberührt und ungeschaut

Steht doch jeder fern und fühlt: alleine.

In der modernen Stadt leben die Menschen dichtgedrängt beieinander. Aber es gibt keine Kommunikation und keine Gemeinschaft, nur namenlose Sehnsucht und stumme Verzweiflung. Das Wort "gewürgt" in der ersten Strophe drückt aus, wie schmerzhaft das Herunterschlucken von Verlangen und Gefühlen ist. Verlangen und Gefühle sind unerwünscht in einer kapitalistischen Gesellschaft. Sie werden als grösstenteils nutzlos und als dem Profit abträglich angesehen. Alfred Wolfensteins Gedicht Das Herz (Pinthus 1996, S. 123) beschreibt die Auswirkungen dieser Sichtweise auf die Menschheit folgendermassen:
Vergessen lag das Herz in unsrer Brust,
Wie lang! ein Kiesel in des Willens Lust,
Nur mit den wasserkühlen spiegelnden Händen
Manchmal berührt, unbewußt.
Einsiedlerisch in sich geschweift so klein,
Nicht nötig für den lückenlosen Stein
Der großen Stadt und für den stählernen Geldthron,
In spitzes Rad griff volles Herz nicht ein.

Das Herz symbolisiert Gefühle. Es liegt vergessen und einsam in unserer Brust, klein und verletzlich, klein wie ein Kieselstein, der auf dem Grund eines Flusses oder Sees liegt. Diese Metapher deutet an, dass der moderne Mensch seine Gefühle überhaupt nicht mehr wahrnimmt. Gefühle werden unterdrückt und vergessen, sodass sie ins Unbewusste hinabsinken. Das kleine, weiche, verletzliche Herz wird kontrastiert mit dem harten, lückenlosen Stein der grossen Stadt, der keinen Raum für Gefühle zulässt. Wolfensteins Wortschöpfung "stählerner Geldthron" weist darauf hin, dass Geld regiert und versinnbildlicht ausserdem Verbindung zwischen Kapitalismus und Krieg, denn die Stahlproduktion ist nicht nur wichtig für die Baubranche sondern auch für die Rüstungsindustrie. 
Krieg ist ein weiteres zentrales Thema in Menschheitsdämmerung. Georg Heyms Gedicht Der Krieg (Pinthus 1996, S. 79) schildert Krieg als personifizierte dunkle, dämonische Macht: „Aufgestanden ist er, welcher lange schlief,/ Aufgestanden unten aus Gewölben tief./ In der Dämmrung steht er gro $\beta$ und unbekannt,/ Und den Mond zerdrückt er in der schwarzen Hand“. Dennoch, Krieg ist kein unausweichliches Schicksal. Kriege sind von Menschen gemacht und können auch von Menschen verhindert werden. Diese Mitverantwortung der Menschheit für Krieg und Frieden ist angedeutet in den folgenden Zeilen: „Und mit tausend hohen Zipfelmützen weit/ Sind die finstren Ebnen flackend überstreut" (S. 79). Das Wort „Zipfelmützen“ weckt Assoziationen mit dem deutschen Michel und mit dem Bild "Der arme Poet" von Carl Spitzweg. Der deutsche Michel ist naiv, autoritätshörig und bequem. Er möchte nicht durch Politik in seiner Ruhe und Behaglichkeit gestört werden. Auch der arme Poet interessiert sich nicht für Politik. Er ist zu stark von seiner Kunst und dem täglichen Überlebenskampf als Künstler in Anspruch genommen. Der Krieg kritisiert politisches Desinteresse als verantwortungslos und strafwürdig, wie die letzte Zeile des Gedichts „Pech und Feuer träufet unten auf Gomorrh" (S. 80) andeutet.

Verantwortung ist auch ein zentrales Thema in Karl Ottens Gedicht Arbeiter! (Pinthus 1996, S. 227-230). Das Gedicht wendet sich in direkter Rede an den Arbeiter als exemplarisch für Millionen von Arbeitern auf der ganzen Welt. Das Gedicht beschreibt die Ausbeutung, Entfremdung und Entmenschlichung des Arbeiters in seiner erzwungenen wirtschaftlichen Abhängigkeit:

Arbeiter! Dich an Rad, Drehbank, Hammer, Beil, Pflug geschmiedeten

Lichtlosen Prometheus rufe ich auf!

Dich mit der rauhen Stimme, dem groben Maul.

Dich Mensch voll Schwei $\beta$, Wunden, Ru $\beta$ und Schmutz

Der du gehorchen mußt.

[...]

Diese Nacht währt lang seit Jahren schwärzeste Finsternis

Ballt sie ihr feuchtes Hemd vor unseren stummen Mund.

[...]

Ihr wißt trotz allem trotz allem

Daß ihr Nummern seid!

Gleichwo: in der Fabrik im Gefängnis im Lazarett in der Kaserne auf dem

Friedhof, 
Ihr seid da für eine Statistik deren Summe, deren Steigen, Fallen, Stocken In jeder Zeitung zu lesen ist. (S. 227-228)

Der Arbeiter ist ein „lichtloser Prometheus". Er ist an Maschinen geschmiedet und muss gehorchen. Er ist ein Geknechteter und Geknebelter (,feuchtes Hemd vor unserem stummen Mund"). Er wird nicht als Mensch wahrgenommen, sondern ist nur eine Nummer in einer Statistik. Das Gedicht prangert die Ausbeutung, Entmündigung und Verdinglichung des Arbeiters im Kapitalismus an. Darüberhinaus weist das Gedicht darauf hin, dass der Arbeiter mitverantwortlich für seine Lage ist:

Ihr schweigt und wartet laßt euch foltern.

Und wie das Herz in immer schwächern Schlägen

Ablaufend Jahr an Jahr gebunden

Schlug es im Takt der Räder Kolben Sägen

Da habt ihr freudig mitgeschunden.

Da hat der Tanz der fauchenden Maschinen

Euch eingelullt und eingeschraubt

Der Eisenfeuergeldgott fuhr aus ihnen

In euer Herz. Er ist es den ihr glaubt. (S. 228-229)

Der Arbeiter wartet, duldet, schweigt. Schlimmer noch, er hat die kapitalistische Ideologie verinnerlicht und ist in seinem Fühlen, Denken und Handeln vom "Eisenfeuergeldgott" fremdgesteuert. Das Gedicht wendet sich an den ausgebeuteten, unterdrückten, entmündigten, entmenschlichten Arbeiter und fordert ihn auf, sich endlich auf sein Menschsein zurückzubesinnen. Auch in Ottens Gedicht nimmt das menschliche Herz einen wichtigen Platz ein. Das Herz symbolisiert wie in Wolfensteins Gedicht menschliche Gefühle. Ausserdem charakterisiert Ottens Gedicht das Herz als Sitz des ethischen Pflichtbewusstseins des Menschen. Das Herz ist synonym mit Menschsein, Erdbewohner sein, eine Seele haben (S. 229). Es steht für ethische Verantwortung und Hoffnung auf Rettung und Erlösung:

Dein Herz verpflichtet dich der Menschheit.

[...]

[...] Du kannst die Menschheit retten!

[...] 
Von deinem Herzen deiner Güte deinem Sein

Von dir allein

Du Sohn der Magd, du Christi Bruder

Hängt ab ob Licht in dieses Meer von Blut und Mördern dringt! (S. 229)

Die oben zitierte Passage aus Ottens Arbeiter! zeigt ein wichtiges Merkmal des Expressionismus auf: Viele expressionistische Schriftsteller nahmen sowohl auf sozialistische als auch auf religiöse Ideen bezug. Otten selbst bezeichnete sich als Anhänger des „messianischen Kommunismus" (Pinthus 1996, S. 357). Auch Bloch bezieht sich in seinen philosophischen Werken auf sozialistische und religiöse Ideen. Besonders stark ausgeprägt ist dieses Merkmal in Blochs Frühwerken Thomas Münzer (1921) und Geist der Utopie (1918, 1923). Der vollständige Titel des ersteren Buches lautet Thomas Münzer als Theologe der Revolution. In ihm bezeichnet Bloch (1921/ 1985, S. 15/ S. 110) Thomas Münzer als "Rebell in Christo" und als "Liebknecht mannigfach verwandt“. Silvia Markun (1977, S. 26) hat bemerkt, dass „messianische Verkündung“ in Blochs Werken „wetterleuchtet“. Markun (1977, S. 26) hat desweiteren darauf hingewiesen, dass Geist der Utopie „[i]n der Sprache des Propheten“ geschrieben ist und dem heutigen Leser einen Einblick in "die authentische Weltanschauung der expressionistischen Generation im Stil wie im Inhalt" eröffnet.

\section{Utopische Vision in ausgewählten Gedichten der Menschheitsdämmerung und in Geist der Utopie}

Johannes R. Bechers Mensch stehe auf (Pinthus 1996, S. 253-258) wendet sich nicht an den Arbeiter, wie Ottens Gedicht, sondern an den Soldaten: „Du Soldat!/ Du Henker und Räuber! Und fürchterlichste der Geißeln Gottes!/ Wann endlich/ - frage ich bekümmert und voll rasender Ungeduld zugleich -/ Wann endlich wirst du mein Bruder sein??" (S. 253) Wie Heyms Der Krieg und Ottens Arbeiter! weist auch Bechers Gedicht auf die Verantwortung der Menschheit für Krieg und Frieden hin. Wie Heyms und Ottens Gedichte beschäftigt sich auch Mensch stehe auf mit der Frage, wie wir Menschen Kriege verhindern können. Bechers Gedicht, das sich in direkter Rede an den Soldaten wendet, stellt die These auf, dass Kriege verhindert werden können,

Wenn

Das mörderische Messer restlos von dir in dir [dem Soldaten] abfällt.

Du vor Gräbern und Feinden waffenlos umkehrst: 


\section{Ein Deserteur! Ein Held! Bedankt! Gebenedeit!}

Zornig du in tausend Stücke das verbrecherische Gewehr zerschmeißt.

Rücksichtslos dich deiner 'verdammten Pflicht und Schuldigkeit' entziehst Und deinen billigen hundsföttischen Dienst höhnisch offen verweigerst allen Ausbeutern, Tyrannen und Lohnherrn. (S. 253)

Der Soldat kann eine innere Wandlung vollziehen. Er kann den Befehl zu töten verweigern und seine Waffe zerbrechen. Das Gedicht entlarvt den Ausdruck „Pflicht und Schuldigkeit" als feige Lüge der Mächtigen, die im Krieg nicht ihr Leben riskieren. Das Adjektiv „hundsföttisch“ kritisiert das Ideal des Soldaten als Befehlsempfänger und verweist indirekt auf ein erstrebenswerteres Ideal: das des mündigen selbständig denkenden und handelnden Subjekts. Die Verwendung der Begriffe "Ausbeuter" und "Lohnherr" deckt die perfide Verbindung zwischen Krieg und Kapitalismus auf. Die Begriffe "umkehrst" und "gebenedeit" verweisen auf Gewaltverzicht und die Geringachtung materieller Güter, wie sie durch Jesus Christus verkörpert werden. Auch die folgenden Zeilen aus Bechers Gedicht enthalten religiöse Begriffe: „Wenn/ Dein [des Soldaten] zerstörerischer Schritt nicht mehr erbarmungslos stampft über die friedlichen Lichtgründe einer kreaturenbeseelten Erde./ Und du dich wütend selbst zermalmst vor deinen glorreichen Opfern am Kreuz./ ...dann dann wirst du mein Bruder sein..." (S. 253).

Sowohl die expressionistischen Dichter als auch Bloch verwendeten den Begriff Brüderlichkeit um Utopie zu umschreiben. Bechers Gedicht bezeichnet die ersehnte Brüderlichkeit zwischen den Menschen ausserdem als Aussöhnung und als ein Sichin-Allen-Erkennen (S. 254): „Dann dann wird gekommen sein jener endliche blendende paradiesische Tag unsrer menschlichen Erfüllung,/ Der Alle mit Allen aussöhnt./ Da Alle sich in Allen erkennen" (S. 254). Bloch verwendet ähnliche Begriffe in Geist der Utopie. Er bezeichnet Utopie als „Allgegenwärtigsein Aller in Allen“ (1923/ 1985, S. 267), als "Brüdergemeinde" (1923/ 1985, S. 346), als "fraternitas auch ohne Vater" (1923/ 1985, S. 267) und als mögliche Erlösung durch das Schaffen einer Gemeinde ohne Herrschaft, „ohne diese Welt, mit Reich“ (1923/ 1985, S. 272). Blochs Vision von Utopie ist sowohl inspiriert von der Idee eines Reiches Gottes auf Erden als auch von Marxs Reich der Freiheit.

Auch Kurt Heynickes Gedicht Gethsemane (Pinthus 1996, S. 76) beschreibt Utopie als Erlösung: „Alle Menschen sind der Heiland./[...]./ Wir sind alle eine Liebe./ Wir sind 
alle tiefes Leid./ Alle wollen sich erlösen“ (S. 76). Den expressionistischen Dichtern war es wichtig hervorzuheben, was die Menschen verbindet, nicht was angeblich trennt (wie z.B. die Zugehörigkeit zu unterschiedlichen Nationen). Heynicke benutzt das Wort "alle" in vier der acht Zeilen, aus denen das Gedicht besteht. Das Personalpronomen "wir" befindet sich dreimal in Heynickes Gedicht. Die Personalpronomen „ich" oder "du“ kommen nicht vor. Die Verwendung des Wortes „Heiland" verweist auf die Bedürftigkeit der Menschheit, ihre Sehnsucht nach Heilung oder Erlösung. Doch kein Aussenstehender könnte die Menschheit heilen oder erlösen. Nur die Menschen selber können dies, indem sie „ins Blaue hinein“ bauen und "das Wahre, Wirkliche, wo das blo $\beta$ Tatsächliche verschwindet" suchen (Bloch, 1923/ 1985, S. 13) - und irgendwann durch Phantasie und Arbeit erschaffen.

Bloch bezeichnet Utopie auch als „das eine Heil“ (Bloch, 1923/ 1985, S. 13).Wie Kurt Heynickes Gedicht Gethsemane handelt auch Ernst Stadlers Gedicht Zwiegespräch (Pinthus 1996, S. 202) von der möglichen Heilung oder Erlösung des Menschen durch sich selbst:

Still, Seele! Kennst du deine eigne Heimat nicht?

Sieh doch: du bist in dir. Das ungewisse Licht,

Das dich verwirrte, war die ewige Lampe, die vor deines Lebens Altar brennt.

Was zitterst du im Dunkel? Bist du nicht selbst das Instrument, Darin der Aufruhr aller Töne sich zu hochzeitlichem Reigen schlingt?

Hörst du die Kinderstimme nicht, die aus der Tiefe leise dir entgegensingt? Fühlst du nicht das reine Auge, das sich über deiner Nächte wildste beugt[...]

Dir selber alles: Fegefeuer, Himmelfahrt und ewige Wiederkehr [...]

Und nichts, was jemals war und wird, das nicht schon immer dein. (S. 202)

Die oben zitierte Passage aus Stadlers Gedicht weckt Assoziationen mit eine Vielzahl Blochscher Ideen: Der Ausdruck "Dir selber alles" verweist auf Blochs Vorstellung der Menschheit als riesiges Reservoir von Möglichkeiten. Bloch zufolge ist der Mensch "die reale Möglichkeit dessen, was in seiner Geschichte aus ihm geworden ist und vor allem mit ungesperrtem Fortschritt noch werden kann" (1959/ 1985, S. 271, hervorgehoben von mir NC). Die letzte Zeile in Stadlers Gedicht „Und 
nichts, was jemals war und wird, das nicht schon immer dein" weckt Assoziationen mit Blochs Idee von Erbe. Dieses Erbe ist Bloch (1923/ 1985, S. 13) zufolge unverloren und unverlierbar, weil utopisches Verlangen eine Grundeigenschaft des Menschen ist und durch nichts und niemanden zerstört werden kann. Stadlers Gedicht enthält ausserdem einen wichtigen Begriff, den Bloch sehr oft benutzte um seine Vision von Utopie zu beschreiben. Wie zuvor erwähnt, bediente Bloch sich häufig religiöser Metaphern in seinem Bemühen utopische Sehnsucht und imaginierte utopische Erfüllung bildhaft zu machen. Er betonte immer wieder, dass er Utopie nicht definieren wolle, denn „ein Genanntes, Herangebrachtes, legt uns bereits auf ein schwaches, eingrenzendes Wort fest" (Bloch 1923/ 1985, S. 247). Er bezeichnete Utopie deshalb auch als "das Eine, Ungenannte, Unnennbare“ (Bloch 1923/ 1985, S. 144). Desweiteren nannte Bloch Utopie "das, was der Knabe in einem Vers aus Goethes Hochzeitslied liegen lie $\beta$, als er aus dem Berg herauskam -'Vergi $\beta$ das Beste nicht!' hatte der Alte zu inm gesagt, aber noch keiner konnte dieses Unscheinbare, tief Versteckte, Ungeheure jemals im Begriff entdecken“ (Bloch 1923/ 1985, S. 244). Ein Begriff jedoch, der diesem Unnennbaren, Unscheinbaren doch tief Versteckten nahe kommt, und den Bloch immer wieder verwendet hat und der auch in Stadlers Gedicht vorkommt, ist der Begriff Heimat. Bloch (1959/ 1985, S. 241) bezeichnete Utopie als , ,jene Freiheit, jene Heimat der Identität, worin sich weder der Mensch zur Welt noch aber auch die Welt zum Menschen verhalten als zu einem Fremden“.

Im Rückblick können sowohl Bloch als auch viele expressionistische Dichter (u.a. Wolfenstein, Otten, und Becher) als Exilschriftsteller bezeichnet werden (sie mussten 1933 aus Deutschland fliehen). Die Nationalsozialisten missbrauchten zahlreiche ihrer Ideen und Begriffe (z.B. Gemeinschaft, Heil, Reich, Heimat) für ihre menschenverachtende Ideologie und Greueltaten. Wie zuvor erwähnt geriet der Expressionismus in den 30er Jahren in Deutschland in den Verdacht dem Aufstieg des Nationalsozialismus Vorschub geleistet zu haben, doch Bloch bestand auf seiner Meinung, der Expressionismus sei zukunftsweisend, weil er den Menschen in den Mittelpunkt stellte. Bloch prophezeite dem Expressionismus sogar eine neue Zukunft: "Das Erbe des Expressionismus ist noch nicht zu Ende, denn es wurde noch gar nicht damit angefangen" (Bloch 1969, S. 59). Auch Kurt Pinthus begriff die deutsche expressionistische Dichtung als Erbe nachfolgender Generationen, von denen er sich wünschte, sie werden den gleichen Mut wie die Generation 1910 bis 1920 aufbringen: „de[n] Mut der Liebe zum gegenwärtigen und zukünftigen Menschen und de[n] Mut zum immerwährenden Versuch in Leben und Dichtung" (Pinthus 1959/ 1996, S. 17). 


\title{
Schlussbetrachtung
}

Geist der Utopie und Menschheitsdämmerung entstanden beide zwischen 1910 und 1920. Beide Werke können stilistisch wie inhaltlich dem Expressionismus zugeordnet werden. Darüberhinaus können die folgenden inhaltlichen Bezugspunkte zwischen Geist der Utopie und Menschheitsdämmerung festgestellt werden:

\begin{abstract}
Musik spielt eine wichtige Rolle in Geist der Utopie und Menschheitsdämmerung. Pinthus gestaltete die Lyrikanthologie Menschheitsdämmerung nach dem Modell einer Symphonie in vier Sätzen. Er betonte die symbolische Bedeutung der sogannnten HumanitätsMelodie von Beethoven für den Expressionismus. In Geist der Utopie widmete Bloch 150 von 350 Seiten der Musik. Wie Pinthus hebt Bloch Beethovens sogenannte Humanitäts-Melodie (in Fidelio) hervor.
\end{abstract}

Geist der Utopie und Menschheitsdämmerung sind beide gesellschaftskritische Werke. Sie kritisieren die Einsamkeit und Entfremdung der Menschheit in der modernen kapitalistischen Gesellschaft. Sie prangern den in der modernen kapitalistischen Gesellschaft vorherrschenden Materialismus als Ursache von spiritueller Leere, Kälte, Phantasiearmut und Gewissenlosigkeit an. Sie kritisieren die Wechselbeziehung zwischen Kapitalismus und Krieg und das weitverbreitete Desinteresse des Durchschnittsbürgers an Politik.

Geist der Utopie und Menschheitsdämmerung beinhalten beide utopische Vision. Sie beschreiben Utopie als Brüderlichkeit und Gemeinschaft, als Aussöhnung und Sich-Erkennen-Aller-In-Allen, als Heilung und Erlösung und als Heimat und Erbe. Beide Werke sind inspiriert und beeinflusst von sozialistischen und religiösen Ideen. Beide Werke beschreiben Utopie mit sozialistischen und religiösen Begriffen.

Geist der Utopie und Menschheitsdämmerung stellen beide den Menschen in den Mittelpunkt und betonen die Verantwortung der Menschheit für ein menschgemässes Leben. Fast ein Jahrhundert nach Erscheinen von Geist der Utopie und Menschheitsdämmerung sind beide Werke erschreckend zeitgemäss. Eine Frage, die sich am Ende dieses Artikels stellt und der nachzugehen mir lohnenswert erscheint, ist: In welchem Ausmass sind die Gedichte der Menschheitsdämmerung und Blochs utopische Philosophie (nicht nur Geist der Utopie, sondern auch Blochs später 
entstandene philosophischen Schriften) anthropozentrisch? Oder schliessen sie eventuell in ihrem Begriff des Ethischen auch den ökologischen Aspekt mit ein?

Finanzielle Förderung: Die Autorin erhielt keine finanzielle Unterstützung für diese Arbeit.

\section{Literaturverzeichnis}

Benjamin, W. (1978). Briefe I. Frankfurt am Main: Suhrkamp.

Bloch, E. (1938/ 1969). Diskussion über Expressionismus. In F. J. Raddatz (Hrsg.), Marxismus und Literatur: Eine Dokumentation in drei Bänden (S. 51-59). Hamburg: Rowohlt Paperback.

Bloch, E. (1971). Pädagogica. Frankfurt am Main: Suhrkamp.

Bloch, E. (1975). "Curriculum Vitae". In L.J. Pongratz (Hrsg.), Philosophie in Selbstdarstellungen. Hamburg: Felix Meiner Verlag.

Bloch, E. (1921/ 1976). Thomas Münzer als Theologe der Revolution. Frankfurt am Main: Suhrkamp.

Bloch, E. (1923/ 1985). Geist der Utopie. Zweite Fassung. Frankfurt am Main: Suhrkamp.

Bloch, E. (1959/ 1985). Das Prinzip Hoffnung. Frankfurt am Main: Suhrkamp.

Bloch, E. (1965/ 1985). Literarische Aufsätze. Frankfurt am Main: Suhrkamp.

Kazin, A. (1976). Introduction. In A. Kazin (Hrsg.), The Portable Blake (S. 1-55). London: Penguin.

Markun, S. (1977). Ernst Bloch in Selbstzeugnissen und Bilddokumenten. Reinbek bei Hamburg: Rowohlt.

Pinthus, K. (Hrsg.) (1996). Menschheitsdämmerung: Ein Dokument des Expressionismus. Hamburg: Rowohlt Taschenbuch.

Serke, J. (1983). Die verbrannten Dichter. Frankfurt am Main: Fischer. 